\title{
On numbers with a unique representation by a binary quadratic form
}

\author{
by
}

\author{
Mariusz SkaŁba (Warszawa)
}

We present a generalization of Davenport's constant and give some number-theoretic application of this notion.

In Section 1 we define the relative Davenport constant $D_{a}(A)$ and prove some basic theorems about it. In particular, we calculate the Davenport constant with respect to any element of a cyclic group and of a $p$-group.

The main result of Section 2 is the following theorem:

Let $F(x, y)$ be a quadratic form with nonsquare discriminant $D$ and conductor $f$. If a natural number $n$, relatively prime to $f$, is uniquely representable by $F$ then

$$
n=r(n) s(n)
$$

where $r(n)$ is a squarefree divisor of $D$ relatively prime to $f, s(n)$ is relatively prime to $D$ and

$$
\Omega(s(n)) \leq D_{[F]^{2}}\left(C(D)^{2}\right)
$$

where $C(D)$ is the corresponding form class group and $\Omega(s(n))$ is the number of prime factors of $s(n)$, counted with multiplicities.

We also obtain an asymptotic formula for the number $N_{F}(x)$ of natural numbers not greater than $x$, relatively prime to $f$ and uniquely representable by the form $F$ :

$$
N_{F}(x)=\left(C_{F}+o(1)\right) \frac{x}{\log x}(\log \log x)^{D_{[F]^{2}}\left(C(D)^{2}\right)-1}
$$

where $C_{F}>0$.

I wish to express my sincere gratitude to Prof. J. Browkin for his help in writing this paper and many valuable suggestions. I am also greatly indebted to the referee for his critical remarks and several improvements.

1. We start with the basic definitions. A sequence $a_{1}, \ldots, a_{k}$ will be called irreducible provided no sum of less than $k$ of its distinct elements 
vanishes. If in addition $a_{1}+\ldots+a_{k} \neq 0$, then this sequence will be called primitive.

For any finite Abelian group $A$ and $a$ in $A$ we define $D_{a}(A)$, the relative Davenport constant of $A$ with respect to $a$, as the greatest integer $k$ with the property that $a$ can be written as the sum of $k$ elements of $A$ forming an irreducible sequence.

For $a=0$ we have $D_{0}(A)=D(A)([3])$.

We need the following easy lemmas:

Lemma 1. Let $A$ be an Abelian group, and $\mathcal{A}=\left(a_{1}, \ldots, a_{k}\right)$ a sequence of its elements. The following conditions are equivalent:

(i) $\mathcal{A}$ is primitive,

(ii) $\mathcal{A}^{\prime}=\left(a_{1}, \ldots, a_{k},-\sum_{i=1}^{k} a_{i}\right)$ is irreducible.

Lemma 2. If $\mathcal{A}=\left(a_{1}, \ldots, a_{k}\right)$ is a maximal primitive sequence in $A$, then every element of $A$ is a sum of elements of $\mathcal{A}$.

First of all we get the following general estimate.

Theorem 1. If $A$ is a finite Abelian group and $a \in A, a \neq 0$, then

$$
\frac{1}{2} D(A) \leq D_{a}(A)<D(A) .
$$

Proof. Let $\mathcal{A}=\left(a_{1}, \ldots, a_{k}\right)$ be an irreducible sequence with sum $a$. Since $a \neq 0$, therefore $\mathcal{A}$ is primitive. From Lemma 1 we see that $\mathcal{A}^{\prime}=$ $\left(a_{1}, \ldots, a_{k},-a\right)$ is also irreducible. Hence $D_{a}(A)<D(A)$.

To prove the estimate from below fix any primitive sequence $\mathcal{A}=\left(a_{1}, \ldots\right.$ $\left.\ldots, a_{k}\right)$ with $k=D(A)-1$. By Lemma 2 we have $a=\sum_{i \in X} a_{i}$ for some $X$.

If $|X| \geq(k+1) / 2$ we define $\mathcal{A}^{\prime}=\left(a_{j}\right)_{j \in X}$. Then $\mathcal{A}^{\prime}$ has sum $a$, is irreducible (even primitive), and therefore

$$
D_{a}(A) \geq \frac{k+1}{2}=\frac{D(A)}{2} .
$$

If $|X|<(k+1) / 2$ we proceed otherwise. Let

$$
Y=\{1, \ldots, k+1\}-X, \quad a_{k+1}=-\sum_{i=1}^{k} a_{i}
$$

and consider the sequence $\mathcal{A}^{\prime \prime}=\left(-a_{j}\right)_{j \in Y}$. It has sum $a$ and is irreducible by Lemma 1, hence

$$
D_{a}(A) \geq k+1-\frac{k+1}{2}=\frac{D(A)}{2} .
$$

Lemma 3. Let $A$ be a finite Abelian group, $B$ a subgroup of $A$ and $a \in B$. Then

$$
D_{a}(A) \leq D_{a}(B) \cdot D(A / B) .
$$


Pr o of. Consider an irreducible sequence $\mathcal{A}=\left(a_{1}, \ldots, a_{n}\right)$ with sum $a$. We may represent the set $\{1, \ldots, n\}$ as the sum of disjoint subsets $A_{1}, \ldots, A_{t}$ $(t \geq 1)$ such that

$$
\forall 1 \leq j \leq t, \quad \sum_{i \in A_{j}} a_{i} \in B \quad \text { and } \quad \forall \emptyset \neq A \varsubsetneqq A_{j}, \quad \sum_{i \in A} a_{i} \notin B .
$$

Then $\left|A_{j}\right| \leq D(A / B)$. If we put

$$
b_{j}=\sum_{i \in A_{j}} a_{i} \quad(j=1, \ldots, t)
$$

then the sequence $b_{1}, \ldots, b_{t}$ has sum $a$ and is irreducible, hence $t \leq D_{a}(B)$ and our assertion follows.

Theorem 2. If $A$ is a finite cyclic group and $a \in A$ then

$$
D_{a}(A)= \begin{cases}|A| & \text { for } a=0, \\ |A|-|A| /|a| & \text { for } a \neq 0\end{cases}
$$

$(|a|$ denotes the order of $a)$.

Proof. Let $A=\mathbb{Z}_{n}(n>1)$. The case $a=0$ is well known. Assume $a \neq 0$. We use Lemma 3 for $B=\langle a\rangle$ :

$$
D_{a}(A) \leq D_{a}(\langle a\rangle) \cdot D(A /\langle a\rangle)=D_{a}(\langle a\rangle) \cdot \frac{n}{|a|} .
$$

Consider the sequence

$$
\mathcal{A}=(-a,-a, \ldots,-a)
$$

with $|a|-1$ terms. $\mathcal{A}$ is primitive and has sum $a$. Hence

$$
D_{a}(\langle a\rangle) \geq|a|-1 .
$$

On the other hand, any irreducible sequence with sum $a$ is primitive $(a \neq 0)$ and hence its length is less than $D(\langle a\rangle)=|a|$. This gives the equality

$$
D_{a}(\langle a\rangle)=|a|-1 .
$$

From the above,

$$
D_{a}(A) \leq n-\frac{n}{|a|} .
$$

To get equality it suffices to construct an irreducible sequence $\mathcal{A}$ with sum $a$ and length $n-n /|a|$. Using an automorphism of $A=\mathbb{Z}_{n}$ if necessary, we may assume that

$$
a=\frac{n}{|a|} \bmod n
$$

and then the sequence

$$
\mathcal{A}=(-1 \bmod n,-1 \bmod n, \ldots,-1 \bmod n)
$$

meets our demand. 
Now we deduce from [5] a formula for $D_{a}(A)$ in case of $p$-groups. We need the following technical definition: Let $A$ be a finite Abelian $p$-group. For any $a \in A$ let

$$
\alpha(a)=p^{n}
$$

where $n$ is the greatest nonnegative integer such that

$$
a=b^{p^{n}}
$$

for $b \in A(\alpha(1)=\infty)$.

Theorem 3. If $A \cong \prod_{i=1}^{r} C_{p^{e_{i}}}$ (where $C_{n}$ denotes the cyclic group of order $n), r \geq 1, e_{i} \geq 1$, then for every nonzero a in $A$ we have

$$
D_{a}(A)=D(A)-\alpha(a) \text {. }
$$

Proof. We write the group $A$ multiplicatively. If $a \neq 1$ and $a=$ $a_{1} \ldots a_{k}$ with $\left(a_{1}, \ldots, a_{k}\right)$ irreducible then Lemma 1 implies that the sequence

$\left(a_{1}, \ldots, a_{k}, a^{-1}\right)$ is irreducible and $\left(a_{2}, \ldots, a_{k}, a^{-1}\right)$ is primitive. Thus the product $\left(1-a_{2}\right) \ldots\left(1-a_{k}\right)\left(1-a^{-1}\right)$ in the group ring $\mathbb{Z}_{p}[A]$ is nonzero and Theorem 2 of [5] implies

$$
\sum_{i=2}^{k} \alpha\left(a_{i}\right)+\alpha\left(a^{-1}\right)<D(A) .
$$

We have $\alpha\left(a_{i}\right) \geq 1$ for $i=2, \ldots, k\left(a_{i} \neq 1\right)$ and $\alpha\left(a^{-1}\right)=\alpha(a)$, therefore

$$
(k-1)+\alpha(a)<D(A),
$$

hence

$$
D_{a}(A) \leq D(A)-\alpha(a) .
$$

To finish the proof it suffices to construct a primitive sequence $\mathcal{A}$ with product $a$ and length $D(A)-\alpha(a)$. Let $b \in A$ be such that

$$
b^{\alpha(a)}=a .
$$

The element $b$ generates a maximal cyclic subgroup of $A$, therefore using possibly an automorphism of $A$ we can write

$$
\exists 1 \leq i \leq r, \quad b=\left(1, \ldots, x_{i}, \ldots, 1\right) \quad \text { where } x_{i} \text { generates } C_{p^{e_{i}}} .
$$

Define

$$
\mathcal{A}=\left(\varepsilon_{1}, \ldots, \varepsilon_{1}, \varepsilon_{2}, \ldots, \varepsilon_{2}, \ldots, \varepsilon_{i}^{-1}, \ldots, \varepsilon_{i}^{-1}, \ldots, \varepsilon_{r}, \ldots, \varepsilon_{r},\left(\prod_{j=1}^{r} \varepsilon_{j}\right) \varepsilon_{i}^{-2}\right)
$$

where for $j \neq i, \varepsilon_{j}:=\left(1, \ldots, x_{j}, \ldots, 1\right)$ appears $p^{e_{j}}-1$ times and $\varepsilon_{i}^{-1}$ $\left(=b^{-1}\right)$ appears $p^{e_{i}}-\alpha(a)-1$ times. 
Corollary. If $A \cong \prod_{i=1}^{r} C_{p^{e_{i}}}, r \geq 1, e_{i} \geq 1$, then

$$
D_{a}(A)= \begin{cases}\sum_{i=1}^{r}\left(p^{e_{i}}-1\right)+1 & \text { for } a=0, \\ \sum_{i=1}^{r}\left(p^{e_{i}}-1\right)+1-\alpha(a) & \text { for } a \neq 0 .\end{cases}
$$

Proof. By Theorem 1 of [5].

2. Let $F(x, y)$ be a binary quadratic form, positive if definite, corresponding to a class $X$ of invertible ideals in an order $\mathcal{O}_{f}$ in a suitable quadratic field $K$. The classical theory of quadratic forms ([1], [2]) shows that if we choose an arbitrary invertible ideal $I$ in $X$ under the unique restriction that in case $X^{2}=E$, the unit class, the ideal $I$ should be ambiguous, i.e. $\bar{I}=I$, then one can choose a $\mathbb{Z}$-basis $a, b$ of $I$ such that

$$
F(x, y)=N(a x-b y) / N(I) \text {. }
$$

Thus we have

$$
F(x, y)=n
$$

with $(x, y)=1$ if and only if there is a principal ideal $A$ with

$$
N(A)=n N(I), \quad A \subseteq I,
$$

which has no rational divisor $>1$. (Actually $A=(a x-b y) \mathcal{O}_{f}$.)

We shall say that $n$ is uniquely representable by the form $F$ provided the ideal $A$ in (1) is unique in the case $X^{2} \neq E$, and unique up to conjugacy in the case $X^{2}=E$.

Lemma 4. Let $X$ be the class of the ideal $I$, assume $(n, f)=1$ and let $A, B$ be distinct, principal and moreover, in the case $X^{2}=E$, nonconjugate ideals satisfying (1). Write

$$
A=I \cdot D_{1} \cdot P_{1} \cdot \ldots \cdot P_{s}, \quad B=I \cdot D_{2} \cdot Q_{1} \cdot \ldots \cdot Q_{t}
$$

where $D_{j}$ are ideals without unramified prime ideal divisors, and $P_{i}, Q_{j}$ are unramified prime ideals in $\mathcal{O}_{f}$; finally, let $a_{i}$ be the class of $P_{i}$ and $b_{j}$ be the class of $Q_{j}$. Then with suitable $i_{j}$ and $r<s$ we have

$$
\left(a_{i_{1}} \cdot \ldots \cdot a_{i_{r}}\right)^{2}=E .
$$

The converse is also true.

Pr o of. Obviously we have $s=t$ and after a suitable regrouping we can assume that $Q_{j}$ either equals $P_{j}$ or is conjugate to it. Assume that the first possibility happens for $j=1, \ldots, w$. Then

$$
b_{j}=a_{j} \quad(j=1, \ldots, w)
$$


and

$$
b_{j}=a_{j}^{-1} \quad(j=w+1, \ldots, s) .
$$

Since $a_{1} \cdot \ldots \cdot a_{s}=b_{1} \cdot \ldots \cdot b_{s}$ we get (2) with $r=s-w$, and it remains to show that $w$ is positive.

Note that $D_{1}, D_{2}$ are both products of distinct ramified prime ideals, since otherwise $A$ resp. $B$ would have a nontrivial rational factor. In view of $N\left(D_{1}\right)=N\left(D_{2}\right)$ this implies $D_{1}=D_{2}$ and so $D_{1} D_{2}$ must be principal.

If $w=0$, then

$$
A B=I^{2} D_{1} D_{2} J \quad \text { where } J \text { is principal, }
$$

showing that $I^{2}$ is principal. But in this case our assumptions give $I=\bar{I}$ and this immediately implies that $A$ and $B$ are conjugate.

To prove the converse we proceed very similarly. After a suitable regrouping we can assume that

$$
\left(a_{1} \cdot \ldots \cdot a_{r}\right)^{2}=E .
$$

Now if we take

$$
D_{2}=D_{1}, \quad Q_{i}=\bar{P}_{i} \quad \text { for } i=1, \ldots, r \quad \text { and } \quad Q_{i}=P_{i} \quad \text { otherwise, }
$$

then $B \neq A$, since equality would imply $P_{1} \cdot \ldots \cdot P_{s}=\bar{P}_{1} \cdot \ldots \cdot \bar{P}_{s}$ and hence $A$ would have a rational divisor $>1$. Moreover, $B \neq \bar{A}$ in the case $X^{2}=E$, since equality would imply $P_{r+1} \cdot \ldots \cdot P_{s}=\bar{P}_{r+1} \cdot \ldots \cdot \bar{P}_{s}$ which also contradicts the assumptions.

The following theorem is an easy consequence of the above lemma and the definition of the relative Davenport constant:

TheOREM 4. Let $F(x, y)$ be a form with nonsquare discriminant $D$ and conductor $f$. If a natural number $n$, relatively prime to $f$, is uniquely representable by $F$ then

$$
n=r(n) s(n)
$$

where $r(n)$ is a squarefree divisor of $D$ relatively prime to $f, s(n)$ is relatively prime to $D$ and

$$
\Omega(s(n)) \leq D_{[F]^{2}}\left(C(D)^{2}\right)
$$

where $[F]$ denotes the class of the form $F$ in the form class group $C(D)$.

Corollary. Let $d$ be a natural number, $d \geq 4$. Moreover, let $f$ be the conductor of the form $F(x, y)=x^{2}+d y^{2}$. If a natural number $x \in[1, \sqrt{3 d})$ is such that $\left(x^{2}+d, f\right)=1$ then either

$$
x^{2}+d=t^{2} \quad \text { for some } t \in \mathbb{N}
$$

or

$$
x^{2}+d=r s
$$


where $r$ is a squarefree divisor of $4 d,(s, 4 d)=1$ and

$$
\Omega(s) \leq D\left(C(-4 d)^{2}\right) .
$$

Pro of. Let $n=x^{2}+d$ for some $x \in[1, \sqrt{3 d})$ and assume that $(n, f)=1$. We have

$$
n<3 d+d=4 d
$$

and

$$
F(x, y) \geq 4 d \quad \text { for }|y| \geq 2,
$$

therefore if $n \neq t^{2}$ then $n$ is uniquely representable by $F$. Now the assertion results from Theorem 4 .

EXAMPLE. Let $d=5005=5 \cdot 7 \cdot 11 \cdot 13$. Since $d$ is squarefree and $d \equiv 1$ $(\bmod 4)$, therefore the conductor $f$ of the form $F(x, y)=x^{2}+5005 y^{2}$ is 1 . Hence for each $x \in[1,122]$,

$$
x^{2}+5005=t^{2} \quad \text { or } \quad x^{2}+5005=r s
$$

where $r \mid 10010,(s, 10010)=1$ and $\Omega(s) \leq 2$.

Theorem 5. Let $F(x, y)$ be a form with discriminant $D<0$ and conductor $f$. For $x \geq 1$, let $N_{F}(x)$ denote the number of natural numbers $n$, not greater than $x$, relatively prime to $f$ and uniquely representable by $F$. Then there exists a positive constant $C_{F}$ such that the following asymptotic equality holds:

$$
N_{F}(x)=\left(C_{F}+o(1)\right) \frac{x}{\log x}(\log \log x)^{D_{[F]^{2}}\left(C(D)^{2}\right)-1} .
$$

Moreover, let

$$
\begin{array}{r}
\bar{N}_{F}(x)=\mid\{n \in \mathbb{N}: n \leq x,(n, f)=1, n \text { is uniquely representable by } F \\
\text { and } \left.\Omega(s(n))=D_{[F]^{2}}\left(C(D)^{2}\right)\right\} \mid .
\end{array}
$$

Then

$$
\lim _{x \rightarrow \infty} \frac{\bar{N}_{F}(x)}{N_{F}(x)}=1 .
$$

Proof. First let us recall some useful definitions. Let $X$ be a set of ideals of the ring $\mathcal{O}_{F}$, and for each ideal $I<\mathcal{O}_{F}$ let $\Omega_{X}(I)$ be the number of prime ideals from $X$ appearing in the decomposition of $I$ into prime factors (counted with multiplicities). If $A$ is a set of prime ideals and

$$
\sum_{\mathfrak{p} \in A} N(\mathfrak{p})^{-s}=a \log \frac{1}{s-1}+g(s) \quad \text { for } \operatorname{Re} s>1
$$

where $g(s)$ is regular in the halfplane $\operatorname{Re} s \geq 1$ then $A$ is called a regular set of prime ideals; the number $a$ is called the Dirichlet density of $A$. 
Lemma 5. Let $\mathcal{O}_{f}$ be an order of an imaginary quadratic field $K$. Let $X$ be a given class of invertible ideals in $\mathcal{O}_{f}$, and $A_{X}$ the set of prime ideals in $X$ relatively prime to $f$. Then the set

$$
\mathcal{A}_{X}:=\left\{\mathfrak{p} \cdot \mathcal{O}_{K}: \mathfrak{p} \in A_{X}\right\}
$$

is regular.

Pr o of. The assertion follows from the proof of Theorem 9.12 of [2], pp. $188-189$.

Let $A$ denote the set of all irreducible sequences of the group $C\left(\mathcal{O}_{f}\right)^{2}$ with product $[I]^{-2}$, where two sequences differing only in the order of terms are considered identical. Let $R$ be the product of all primes dividing $D$ and relatively prime to $f$, and $r$ a fixed divisor of $R$. Moreover, let $\mathcal{R}$ be the product of prime ideals of $\mathcal{O}_{f}$, dividing $r$.

For each $\mathcal{A}=\left(\alpha_{1}, \ldots, \alpha_{k}\right) \in A$ we define

$$
\begin{aligned}
\mathcal{A}(r)=\left\{\mathcal{B}=\left(\beta_{1}, \ldots, \beta_{k}\right)\right. & : \beta_{i} \in C\left(\mathcal{O}_{f}\right), \beta_{i}^{2}=\alpha_{i} \\
& \text { for } \left.i=1, \ldots, k \text { and } \prod_{i=1}^{k} \beta_{i}=[I]^{-1}[\mathcal{R}]^{-1}\right\} .
\end{aligned}
$$

First we prove that for any $\mathcal{A} \in A$,

$$
\mathcal{A}(r) \neq \emptyset .
$$

Let $\mathcal{B}^{\prime}=\left(\beta_{1}^{\prime}, \ldots, \beta_{k}^{\prime}\right)$ be an arbitrary sequence of elements of $C\left(\mathcal{O}_{f}\right)$ such that $\beta_{i}^{\prime 2}=\alpha_{i}$ for $i=1, \ldots, k$. Since

$$
\prod_{i=1}^{k} \beta_{i}^{\prime 2}=\prod_{i=1}^{k} \alpha_{i}=\left([I]^{-1}[\mathcal{R}]^{-1}\right)^{2} \quad\left([\mathcal{R}]^{2}=1\right),
$$

there exists $\beta^{\prime} \in C\left(\mathcal{O}_{f}\right)$ such that $\beta^{\prime 2}=1$ and

$$
\beta^{\prime} \cdot \prod_{i=1}^{k} \beta_{i}^{\prime}=[I]^{-1}[\mathcal{R}]^{-1} .
$$

Hence

$$
\mathcal{B}:=\left(\beta^{\prime} \beta_{1}^{\prime}, \beta_{2}^{\prime}, \ldots, \beta_{k}^{\prime}\right) \in \mathcal{A}(r),
$$

which ends the proof of $(*)$.

Define

$$
\mathcal{U}=\{n \in \mathbb{N}:(n, f)=1, n \text { is uniquely representable by } F\}
$$

and for each $r \mid R$ let

$$
\mathcal{U}(r)=\{n \in \mathcal{U}: r(n)=r\}
$$


(with $r(n)$ from Theorem 4). Clearly

$$
\mathcal{U}=\bigcup_{r \mid R} \mathcal{U}(r) \text {. }
$$

Hence

$$
N_{F}(x)=\sum_{r \mid R} N_{F}^{(r)}(x)
$$

where

$$
N_{F}^{(r)}(x):=|\{n \in \mathcal{U}(r): n \leq x\}| .
$$

We first obtain an asymptotics for $N_{F}^{(r)}(x)$ at a fixed $r \mid R$ and then use $(* *)$. Let

$$
\begin{gathered}
h=\left|C\left(\mathcal{O}_{f}\right)\right|, \quad C\left(\mathcal{O}_{f}\right)=\left\{\gamma_{1}, \ldots, \gamma_{h}\right\}, \\
\Pi_{i}=\left\{\mathfrak{p} \cdot \mathcal{O}_{F}: \mathfrak{p} \text { a prime ideal of } \mathcal{O}_{f},(N(\mathfrak{p}), f)=1 \text { and }[\mathfrak{p}]=\gamma_{i}\right\} .
\end{gathered}
$$

For each sequence $\mathcal{B}=\left(\beta_{1}, \ldots, \beta_{n}\right)$ of elements of $C\left(\mathcal{O}_{f}\right)$ let

$$
\Omega_{\Pi_{i}}(\mathcal{B}):=\left|\left\{j \in 1, \ldots, n: \beta_{j}=\gamma_{i}\right\}\right| \text {. }
$$

We define

$$
\begin{aligned}
\mathcal{J}(r)=\bigcup_{\mathcal{A} \in A} \bigcup_{\mathcal{B} \in \mathcal{A}(r)}\left\{J \cdot \mathcal{O}_{K}: J<\mathcal{O}_{f},(N(J), f)=1\right. \text { and } & \\
& \left.\Omega_{\Pi_{i}}\left(J \cdot \mathcal{O}_{K}\right)=\Omega_{\Pi_{i}}(\mathcal{B}) \text { for } i=1, \ldots, h\right\} .
\end{aligned}
$$

From the above definitions and Lemma 4 it follows that the map $\mathcal{N}: \mathcal{J}(r) \rightarrow$ $\mathbb{N}$ given by the formula

$$
\mathcal{N}(J):=N(J) \cdot r
$$

maps $\mathcal{J}(r)$ onto $\mathcal{U}(r)$ and moreover, for all but finitely many $n \in \mathcal{U}(r)$,

$$
\left|\mathcal{N}^{-1}(n)\right|= \begin{cases}1 & \text { if } X^{2} \neq E \\ 2 & \text { if } X^{2}=E\end{cases}
$$

By Lemma 5 and Proposition 9.6 in Ch. 9 of [4],

$$
|\{J \in \mathcal{J}(r): \mathcal{N}(J) \leq x\}|=\sum_{\mathcal{A} \in A} \sum_{\mathcal{B} \in \mathcal{A}(r)}\left(C_{\mathcal{B}}+o(1)\right) \frac{\frac{x}{r}}{\log \frac{x}{r}}\left(\log \log \frac{x}{r}\right)^{l(\mathcal{B})-1},
$$

hence by $(*)$ and the definition of the relative Davenport constant,

$$
|\{J \in \mathcal{J}(r): \mathcal{N}(J) \leq x\}|=\left(C_{r}^{\prime}+o(1)\right) \frac{x}{\log x}(\log \log x)^{D_{[I]^{2}}\left(C\left(\mathcal{O}_{f}\right)^{2}\right)-1} .
$$

From $(* * *)$ and the above formula,

$$
N_{F}^{(r)}(x)=\left(C_{r}+o(1)\right) \frac{x}{\log x}(\log \log x)^{D_{[I]^{2}}\left(C\left(\mathcal{O}_{f}\right)^{2}\right)-1} .
$$


To obtain the first part of the assertion of Theorem 5 it suffices to use $(* *)$. The second part, concerning the function $\bar{N}_{F}(x)$, is now obvious.

\section{References}

[1] Z. I. Borevich and I. R. Shafarevich, Number Theory, Nauka, Moscow 1985 (in Russian).

[2] D. A. Cox, Primes of the Form $x^{2}+n y^{2}$, Wiley-Interscience, New York 1989.

[3] H. Davenport, in: Proceedings of the Midwestern Conference on Group Theory, Ohio State University, April 1966.

[4] W. Narkiewicz, Elementary and Analytic Theory of Algebraic Numbers, PWN, Warszawa 1990.

[5] J. E. Olson, A combinatorial problem on finite Abelian groups, I, II, J. Number Theory 1 (1969), 8-10, 195-199.

INSTITUTE OF MATHEMATICS

WARSAW UNIVERSITY

BANACHA 2

02-097 WARSZAWA, POLAND

Received on 10.3.1992

and in revised form on 5.6.1992 